\title{
Serum Growth Hormone Levels and Size of Pituitary Tumour in Untreated Acromegaly
}

\author{
A. D. WRIGHT,* M.B., M.R.C.P. ; M. S. F. MCLACHLAN, $\dagger$ M.R.C.P.ED., D.M.R.D., F.F.R. \\ F. H. DOYLE, $\ddagger$ M.B., D.M.R.D., F.F.R. ; T. RUSSELL FRASER,§ M.D., F.R.C.P.
}

British Medical fournal, 1969, 4, 582-584

\begin{abstract}
Summary : Serum levels of growth hormone were measured in 84 untreated acromegalic patients. The range of mean level during a glucose tolerance test was 8 to $1,860 \mathrm{ng} . / \mathrm{ml}$. and was lognormally distributed. Mean level was reproducible and correlated moderately well with maximum sellar area on lateral tomography. The hormone level was more responsive to oral glucose and insulin-induced hypoglycaemia in patients with smaller tumours. Attention is drawn to limitations of plain radiographs in assessing the size of a pituitary tumour. Suprasellar tumour extensions may be present in patients without visual field defects.
\end{abstract}

\section{Introduction}

Acromegaly is characterized by excessive secretion of growth hormone, usually by an adenoma of somatotropic cells of the anterior pituitary. The level of the hormone in serum is raised and fails to suppress normally after oral or intravenous glucose (Glick et al., 1965). In some acromegalic patients, however, circulating levels of growth hormone are partially suppressed by glucose or may even show a "paradoxical "ris: during a glucose tolerance test-for example, Glick et al. (1965), Cryer and Daughaday (1969), and Burger and Catt (1969). The serum level of the hormone and the size of the pituitary adenoma vary widely in any series of acromegalic patients. Sönksen et al. (1967) found an association between the size of the pituitary fossa and the plasma level of growth hormone. Forrest et al. (1965) suggested that sellar size and serum level of the hormone were not correlated.

We have studied the relationship of sellar size to the serum level of growth hormone and its responsiveness to oral glucose and insulin-induced hypoglycaemia in 84 untreated acromegalic patients. The influence of age, sex, and duration of the disease was examined. In 22 untreated patients measurements of serum growth hormone were repeated within 13 months. The reproducibility of these measurements has been analysed.

\section{Patients Studied}

All patients had obvious clinical features of acromegaly, and their serum growth hormone levels failed to suppress below $5 \mathrm{ng} . / \mathrm{ml}$. during a glucose tolerance test. Lateral tomograms of the pituitary fossa were taken routinely on every patient. Forty-six were men (mean age 45, range 22-65 years) and 38 were women (mean age 50, range 25-64 years). The onset of the disease was estimated from the clinical history and whenever possible by inspection of previous photographs. The disease started before 18 years of age in three patients, in one of whom excessive growth in height had occurred. The duration of the disease could not be assessed in nine patients.

\footnotetext{
* Registrar.

† Senior Registrar.

¥ Reader in Diagnostic Radiology.
S Professor of Clinical Endocrinology

Departments of Medicine and Diagnostic Radiology, Hammersmith Hospital and Royal Postgraduate Medical School, London W.12.
}

\section{Methods}

Serum growth hormone was measured by a modification of the method of radioimmunoassay described by Hartog et al. (1964). Results were expressed as ng./ml., using the Medical Research Council reference standard " $A$ " for human growth hormone. For every assay run during this study samples from reference pools of sera were also assayed. The coefficients of variation for different assays of the reference sera varied from 17 to $23 \%$.

Growth hormone was measured in serum taken after an overnight fast and at intervals of 30 minutes for two to two and a half hours after $50 \mathrm{~g}$. of oral glucose. The area under the points was measured and divided by the time in hours to give the mean level of the hormone during the glucose tolerance test. The level at one hour was expressed as a percentage of the fasting level to give an estimate of the responsiveness to oral glucose. This information is available in 81 patients.

In 49 patients serum levels of the hormone were measured after intravenous insulin in a dose of $0.3 \mathrm{unit} / \mathrm{kg}$. body weight (Fraser et al., 1962). The level at one hour expressed as a percentage of the fasting level was taken as a measure of the responsiveness to insulin-induced hypoglycaemia. The serum level of the hormone was defined as unresponsive to either oral glucose or insulin-induced hypoglycaemia when the one-hour level was within $\pm 50 \%$ of the fasting level.

The size of pituitary tumour was estimated from lateral tomograms of the sella. Plain films of the pituitary fossa often fail to show large downward extensions of tumour into the sphenoid sinus, and tumour size may be grossly underrated (Fig. 1). The tomographic cut which showed the greatest sellar expansion was selected. This radiograph was projected on to white cardboard and a tracing made of sellar outline. A straight line joining the tuberculum sellae and the tip of the dorsum formed the upper limit of the outline. Each tracing was cut out, weighed, and compared with a standard area. Corrections were made for radiographic and optical magnification.

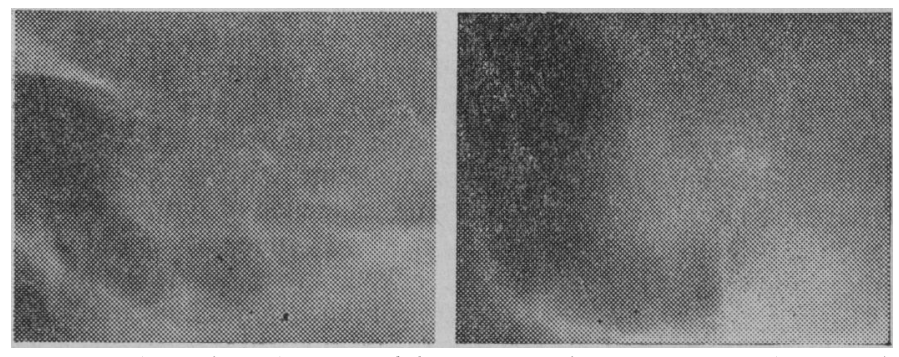

FIG. 1.-Lateral radiograph (left) and midline tomogram (right) of pituitary fossa of a 63-year-old man with acromegaly. On the plain film only a small tumour was suspected. The calcification is in carotid siphons. The tomogram shows that the tumour extends inferiorly as far as the floor of the sphenoid sinus.

Maximum lateral gland area could be measured in 28 patients in whom pneumoencephalography was performed as described by McLachlan et al. (1969). Seven patients showed upward extensions of the pituitary tumour, and in two the superior 
aspect of the tumour was sunken within the sella (Figs. 2 and 3). The extent of these deviations above or below the line drawn between the tuberculum and the tip of the dorsum was measured in the same way as lateral sellar area. By adding or subtracting these deviations the maximum lateral gland area was derived. This was compared with serum growth hormone levels in these 28 patients.

\section{Results}

Maximum lateral area of the sella varied nearly tenfold, from 79 to 727 sq. mm., in the 84 patients. Those who had had the disease for more than 15 years had significantly larger tumours than those who had had the disease for less than five years (Fig. 4). Sellar area was independent of age and sex.

Mean serum growth hormone during the glucose tolerance test varied over 200 -fold, from 8 to $1,860 \mathrm{ng} . / \mathrm{ml}$., in the 84 patients and was lognormally distributed. It was not correlated with age, sex, or duration of disease. In the 22 patients in whom repeat measurements of the mean serum level during a glucose tolerance test were made within 13 months the two measurements were highly correlated (Fig. $5, \mathrm{r}=0 \cdot 89$ ).

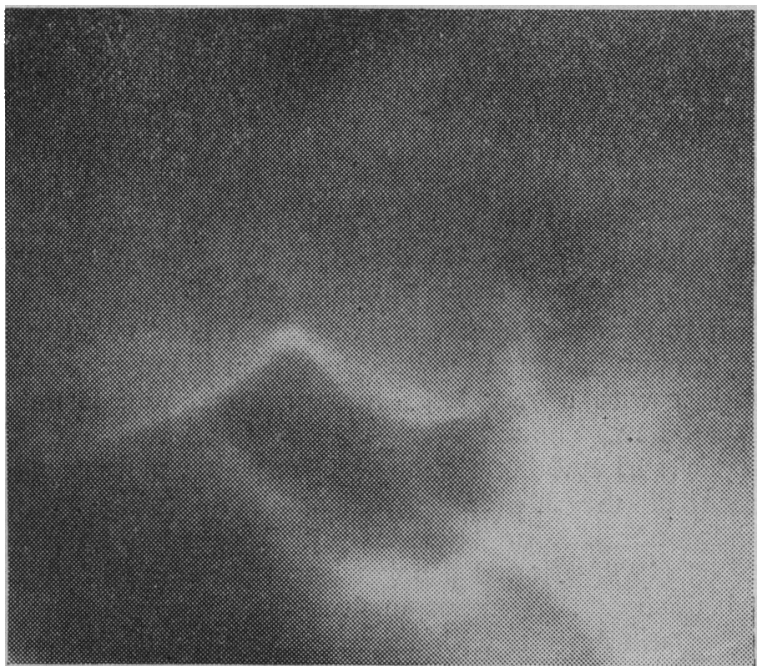

FIG. 2.-Combined pneumoencephalography and lateral tomography demonstrate a large upward extension of tumour in a 46-year-old man with acromegaly. Seven of the 28 patients similarly investigated had significant upward extensions. This patient was the only one with a visual field defect.
Mean serum growth hormone level was moderately well correlated with maximum lateral sellar area $(\mathrm{r}=0.47 ; \mathrm{P}<0.001$. Fig. 6). The correlation was slightly better in females $(r=$ $0.55 ; \mathrm{P}<0.002)$ than in males $(\mathrm{r}=0.39 ; \mathrm{P}<0.003)$. In the 28 patients in whom pneumoencephalography was carried out the correlation of mean serum growth hormone with maximum lateral area at the gland was better than that with lateral area of the sella $(r=0.70$ compared with 0.55$)$, but the difference was not statistically significant $(0.4>\mathrm{P}>0.3)$.

In the majority of patients $(68 / 81 ; 84 \%)$ the serum growth hormone level was unresponsive to glucose. A minority (12/ $81 ; 15 \%$ ) showed a "paradoxical " rise in serum level at one hour, and in only one patient was the level suppressed to below $50 \%$ of the fasting level. Further analysis showed that responsiveness to glucose was related to tumour size. Lateral sellar area was less than 350 sq. $\mathrm{mm}$. in 12 of the 13 patients whose growth hormone levels were responsive to glucose (Fig. 7).

Serum growth hormone level did not respond to insulininduced hypoglycaemia in 36 of the 49 patients tested (74\%). Ten patients $(20 \%)$ responded normally with a greater than $50 \%$ increase in the level at one hour compared with the fasting level. In three patients the one-hour level showed a "paradoxical" suppression to less than $50 \%$ of the fasting level.

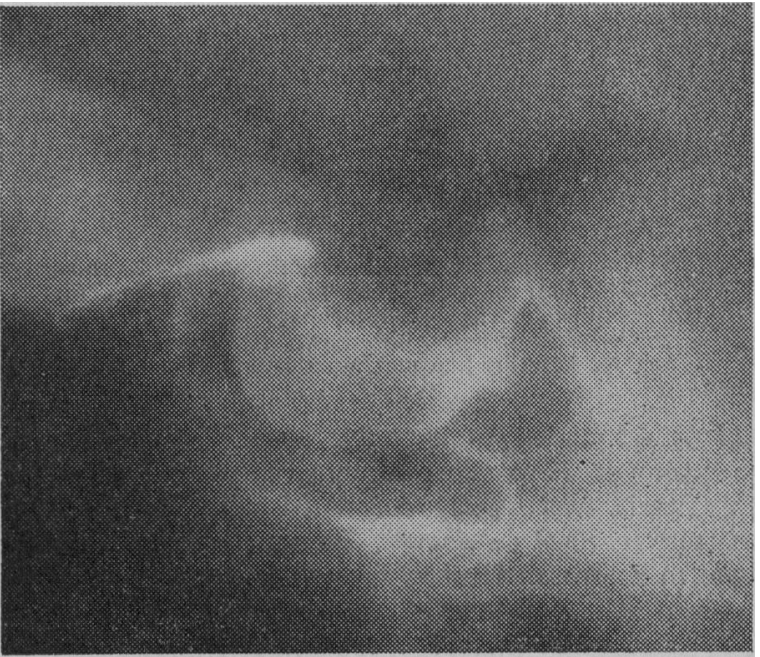

FIG. 3.-This 53-year-old man was one of two patients in whom the upper surface of the tumour was sunken below the expected position of the diaphragm. Neither patient had any clinical episode that could be interpreted as pituitary apoplexy or tumour infarction.

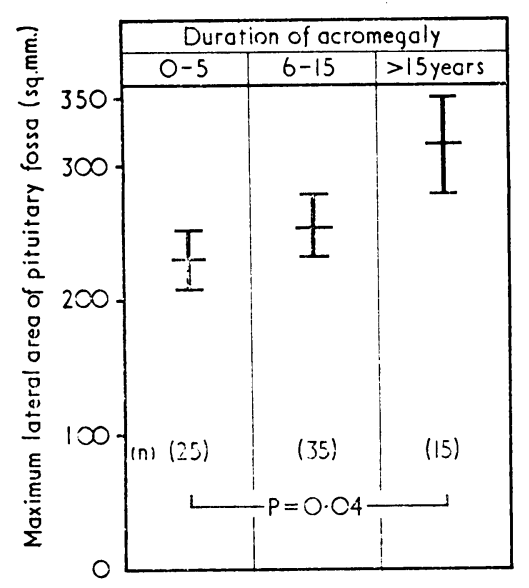

FIG. 4

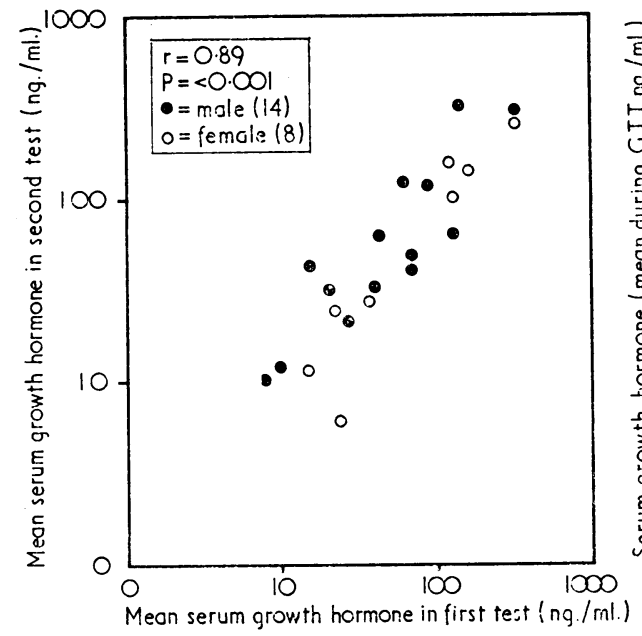

Fig, 5

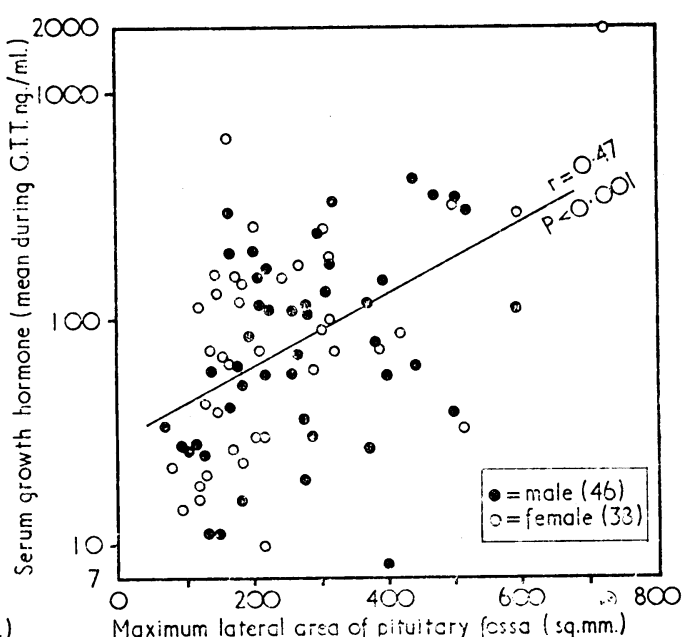

FIG. 6

FIg. 4-Maximum lateral sellar area (mean + S.E7.) in untreated acromegalic patients divided into three groups according to duration of FIG. 4.- disease. Fis. 5.-Re - Relow hormone level during an oral glucose tolerance tolernnce test. Fig. 6.-Relationship of maximum lateral sellar area to the mean serum growt 
Patients showing a response to insulin-induced hypoglycaemia had smaller fossae than those who did not respond (Fig. 7).

Responsiveness to hypoglycaemia and to oral glucose were related (see Table). Of the eight patients responsive to glucose five $(63 \%)$ were responsive to insulin-induced hypoglyzaemia. Of the 40 patients unresponsive to glucose, only

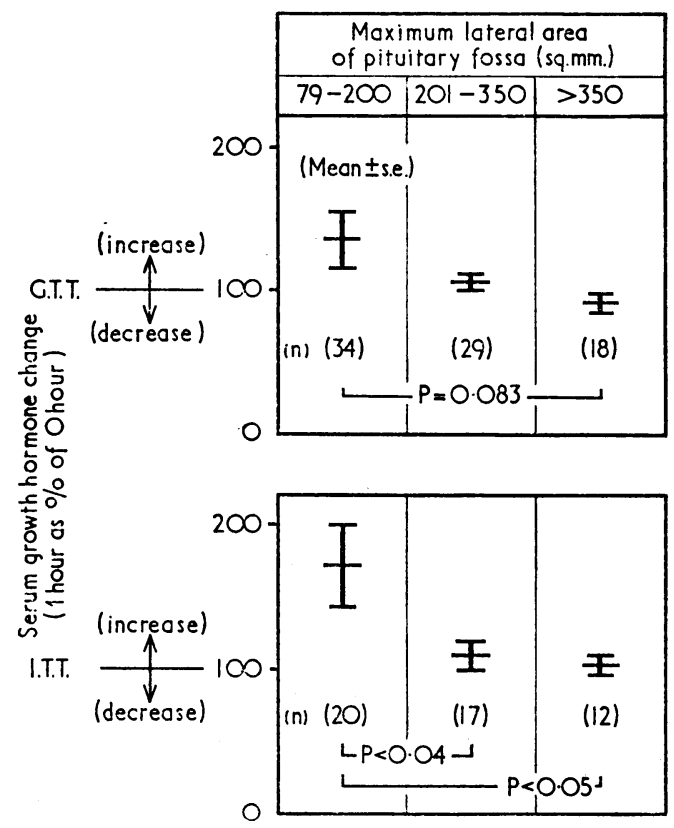

FIG. 7.-Responsiveness of serum growth hormone levels ( 1 hour as $\% 0$ hour) to glucose ( 81 patients)
and insulin-induced hypoglycaemia ( 49 patients). The mean \pm S.E. response is shown for three different groups according to maximum lateral sellar area.

six $(15 \%)$ responded to hypoglycaemia $(P<0 \cdot 01)$. For the whole group of 49 patients responsiveness to insulin-induced hypoglycaemia was weakly correlated with responsiveness to oral glucose $(r=0.27 ; P=0.03)$. Responsiveness did not appear to relate to the mean level of growth hormone.

Response of Serum Growth Hormone Levels to Oral Glucose Related to the Response to Insulin-induced Hypoglycaemia in 49 Untreated Acromegalic Patients

\begin{tabular}{|c|c|c|c|c|}
\hline & & \multicolumn{3}{|c|}{ Response to Oral Glucose } \\
\hline & & Rise (8) & $\mathrm{Nil}(40)$ & Fall (1) \\
\hline $\begin{array}{l}\text { Response to } \\
\text { hypoglycaemia }\end{array}$ & $\begin{array}{l}\text { Rise (11) } \\
\text { Nil (36) } \\
\text { Fall (2) }\end{array}$ & $\begin{array}{l}5 \\
2 \\
1\end{array}$ & $\begin{array}{r}6 \\
33 \\
1\end{array}$ & $\overline{1}$ \\
\hline
\end{tabular}

Nil response: one-hour level within $\pm 50 \%$ of fasting level.

\section{Discussion}

Though sellar and gland volume can be predicted with reasonable accuracy from anteroposterior and lateral radiographs in the normal subject (Di Chiro and Nelson, 1962; McLachlan et al., 1968), we have not attempted to measure tumour volume from radiographs. Plain $x$-ray films of the skull provide an inadequate picture of the size of a pituitary tumour. Lateral tomography ensures that large extensions into the sphenoid sinus are not overlooked but does not define the upper limit of a tumour. The combination of pneumoencephalography and tomography may show unexpected suprasellar extensions of a pituitary tumour. Seven out of 28 patients in whom the combined procedure was used had significant extensions above the expected position of the diaphragma sellae. Only one of these had a visual field defect. In our view the maximum lateral gland area measured by combining pneumoencephalo- graphy with tomography provides the best readily achievable estimate of tumour size.

The highly significant correlation between the maximum lateral area of the sella and the mean serum growth hormone during a glucose tolerance test in patients with untreated acromegaly suggests that growth hormone secretion is proportional to the size of the pituitary tumour. This is in agreement with the observations of Sönksen et al. (1967), who estimated fossa volume from anteroposterior and lateral radiographs, tomography being used when necessary. This measurement was significantly correlated with serum growth hormone assayed during intravenous glucose tolerance tests in 16 patients with acromegaly. These authors found an inverse relationship between fossa volume and age, but this correlation was heavily weighted by two young patients with very large tumours. In our much larger series the lateral fossa area and age are not correlated. Fossa area is, however, related to duration of disease (Fig. 4).

The responsiveness of serum growth hormone level to changes in blood sugar is probably mediated via the hypothalamus (Glick et al., 1965). Responsiveness therefore depends on an intact pathway between hypothalamus and anterior pituitary, and on anterior pituitary cells capable of responding to stimuli from the hypothalamus. The failure of growth hormone level to respond to changes in blood glucose in patients with acromegaly can be explained in two ways. Firstly, the pathway between hypothalamus and anterior pituitary is interrupted. Secondly, the pathway is intact but there are no pituitary cells capable of responding to the stimuli. In acromegalics whose growth hormone levels remain responsive to changes in blood glucose the pathway can be presumed to be intact. Stimuli from the hypothalamus could be effected either via tumour cells or through normal anterior pituitary cells.

We have shown that size of tumour is related to responsiveness of growth hormone level to changes in blood sugar. Thus no patient with a very large tumour (maximum lateral area $>350$ sq. $\mathrm{mm}$.) showed a response to insulin-induced hypoglycaemia and only one showed a response to oral glucose. Thirteen of the 37 (35\%) patients with smaller tumours responded normally or "paradoxically" to hypoglycaemia ; 12 out of $64(19 \%)$ showed a normal or "paradoxical " response to oral glucose. The unresponsiveness of serum growth hormone levels in patients with acromegaly reflects either pituitary stalk section or loss of somatotropic cells responsive to stimuli from the hypothalamus. The larger the tumour the more likely are these to occur.

We are grateful to Miss Susan Russell for her help. The radioimmunoassay of growth hormone was supported by a grant from the Medical Research Council.

Burger, H. G., and Catt, K. J. (1969). Lancet, 1, 1213.

Cryer, P. E., and Daughaday, W. H. (1969). fournal of Clinical Endocrinology, 29, 386.

Di Chiro, G., and Nelson, K. B. (1962). American fournal of Roentgenology, 87, 989.

Forrest, A. P. M., et al. (1965). Proceedings of the Royal Society of Medicine, 58,475.

Fraser, R., Joplin, G. F., Opie, L. H., and Rabinowitz, D. (1962). Fournal of Endocrinology, 25, 299.

Glick, S. M., Roth, J., Yalow, R. S., and Berson, S. A. (1965). Recent Progress in Hormone Research, 21, 241.

Hartog, M., Gaafar, M. A., Meisser, B., and Fraser, R. (1964). British Medical fournal, 2, 1229.

McLachlan, M. S. F., Edwards, C. R. W., and Lavender, J. P. (1969). In preparation.

McLachlan, M. S. F., Williams, E. D., Fortt, R. W., and Doyle, F. H. (1968). British fournal of Radiology, 41, 323.

Sönksen, P. H., et al. (1967). Fournal of Clinical Endocrinology, 27, 1418. 